\title{
Editorial
}

\section{Inverse Synthetic Aperture Radar}

\author{
Marco Martorella, ${ }^{1,2}$ John Homer, ${ }^{3}$ James Palmer, ${ }^{4}$ Victor Chen, ${ }^{5}$ Fabrizio Berizzi, ${ }^{1,2}$ \\ Brad Littleton, ${ }^{6}$ and Dennis Longstaff ${ }^{1}$ \\ ${ }^{1}$ The school of ITEF, The University of Queensland, Brisbane 4072, Australia \\ ${ }^{2}$ Department of Information Engineering, University of Pisa, Via G. Caruso 16, 56122 Pisa, Italy \\ ${ }^{3}$ School of Information Technology \& Electrical Engineering, University of Queensland, Brisbane 4072, Australia \\ ${ }^{4}$ Radar Modelling \& Analysis Group, Electronic Warfare \& Radar Division, Defence Science \& Technology Organisation, \\ P.O. Box 1500, Edinburgh 5111, UK \\ ${ }^{5}$ Naval Research Laboratory, 4555 Overlook Ave., SW Washington, DC 20375, USA \\ ${ }^{6}$ Centre for Quantum Computer Technology, School of Physical Sciences, University of Queensland, Brisbane 4072, Australia
}

Received 2 March 2006; Accepted 2 March 2006

Copyright (c) 2006 Marco Martorella et al. This is an open access article distributed under the Creative Commons Attribution License, which permits unrestricted use, distribution, and reproduction in any medium, provided the original work is properly cited.

\section{Introduction to ISAR}

Inverse synthetic aperture Radar (ISAR) is a powerful signal processing technique that can provide a two-dimensional electromagnetic image of an area or target of interest. Being radar based, this imaging technique can be employed in all weather and day/night conditions. ISAR images are obtained by coherently processing the received radar echoes of transmitted pulses. Commonly, the ISAR image is characterised by high resolution along both the range and crossrange directions. High resolution in the range direction is achieved by means of large bandwidth transmitted pulses, whereas high cross-range resolution is obtained by exploiting a synthetic antenna aperture. In ISAR, the synthetic aperture is generated by motion of the target as well as possibly by motion of the radar platform. In contrast, the related imaging technique of Synthetic aperture radar (SAR) has its synthetic aperture generated by means of radar platform motion only.

Initially, the name ISAR was derived from SAR by simply considering a radar-target dynamic where the radar platform was fixed on the ground and the target was moving around. Today, however, it is understood that the basis of the difference between SAR and ISAR lies in the noncooperation of the ISAR target. Such a subtle difference has led in the last decades to a significant separation of the two areas. The noncooperation of the target introduces the main problem of not knowing the geometry and dynamic of the radar-target system during the coherent integration time. Such a limitation leads to the use of blind radial motion compensation (image autofocusing) and image formation processing that must deal with highly nonstationary signals.

The SAR community is very large and the areas of interest within SAR grow steadily each year. The ISAR community is much smaller, in comparison, and it is often difficult to bring together world leaders in this sector. This special issue aims to gather the latest novelties in ISAR in order to provide an updated reference for current and future research in this area. This has involved a comprehensive peer review process to guarantee technical novelty and correctness. As discussed below, the presented papers, six in total, are equally divided amongst the three primary areas of ISAR research, namely: motion compensation (or image autofocusing), image formation, and target classification/recognition. Whereas the first two areas are devoted to the reconstruction of the ISAR image, the latter concerns the use of the ISAR image for target recognition-one of the principle motivations for ISAR development.

\section{Motion compensation}

Motion compensation is the first step in the ISAR image reconstruction chain. Image focus and clarity strongly depend on the accuracy of motion compensation. Often referred to as image focusing or image autofocusing (blind data driven motion compensation), the motion compensation problem has been largely addressed since the beginning of ISAR. Several algorithms have been provided that accomplish motion compensation. Nonparametric algorithms such as prominent point processing (PPP) and phase gradient algorithm 
(PGA) often, in the past, have been applied in ISAR imaging, largely because they do not need a signal model assumption. More recently, several other nonparametric methods, such as the maximum likelihood- (ML-) based technique and the joint time-frequency analysis (JTFA) technique, have been proposed and are proving to be relatively effective. On the other hand, parametric approaches, such as image-entropy or image contrast-based algorithms, are attracting increased attention due to the potential enhancements they can provide over nonparametric approaches.

In this special issue, two papers are presented which address the problem of motion compensation. The first, written by Martorella et al., concerns a general extension of two parametric algorithms, namely, the image contrast based-algorithm (ICBA) and image-entropy-based algorithm (IEBA). A second-order polynomial phase model is often used as the parametric model for motion compensation in algorithms such as the ICBA and the IEBA. Often such a model does not prove to be accurate enough, due to irregular target motions, such as in the cases of fast manoeuvring targets or sea-driven target angular motions in rough sea surface conditions. Motivated by this, researchers, such as those of the Martorella et al. paper, are employing high-order polynomial phase models to achieve accurate image focussing. However, estimation of the required polynomial coefficients (via solving of an optimisation problem) is typically sensitive to the cost function (image contrast or entropy) and the iterative-search technique employed. In particular, solutions provided by classic iterative techniques, such as Newton, quasi-Newton, steepest descent, or gradient, are generally unsuitable due to the multimodal characteristics of the cost function (which become more severe as the number of polynomial coefficients increases). To avoid such convergence problems Martorella et al. consider a geneticbased iterative technique, which they apply to the estimation/optimisation of a third-order polynomial phase model.

The second paper, written by Yau et al., also addresses the multimodal-related convergence difficulties associated with many parametric-based motion compensation approaches. This paper proposes to overcome the difficulties by decoupling the estimation of the first- and higher-order polynomial coefficients. This is accomplished via an iterative twostage approach; first a range-profile cross-correlation step is applied to estimate the first-order coefficient, and then a subspace-based technique, involving eigenvalue decomposition (EVD) or singular value decomposition (SVD), is applied to estimate the higher-order coefficients. The potential benefits of this two-stage approach arise because the optimisation process is implemented over two lower-dimensional spaces, thereby enhancing the likelihood of convergence to a globally optimal solution.

\section{Image formation}

After motion compensation, the received signal is processed to form the ISAR image. The classic way of forming an ISAR image involves a two-step process. The first step concerns the range compression (or range focussing). Here, either the received time-domain signals are compressed by means of matched filters or the received multifrequency signals are compressed via the inverse Fourier transform-to produce complex range profiles. It is worth pointing out that in some cases the range compression is achieved before the motion compensation. The second step consists of cross-range compression (azimuth compression). The fastest and simplest way of obtaining cross-range compression is by means of a Fourier transform. In ISAR scenarios, where the target is moving smoothly with respect to the radar and when the integration time is short enough, the Fourier transform represents the most effective solution. Nevertheless, in ISAR scenarios with fast manoeuvring targets or sea-driven motioned ships or with the requirement of high resolution, the effectiveness of the Fourier approach is strongly limited. For this reason, several other techniques have been proposed in the last decades, such as the JTFA, the range-instantaneousDoppler (RID), the enhanced image processing (EIP) techniques, tomography-based techniques and super-resolution techniques, such as the CLEAN technique, and the Capon technique among others.

In this special issue, the paper by Djurovic et al. proposes a novel image formation (cross-range compression) technique based on the use of the polynomial Fourier transform (PFT) for enhancing the ISAR image quality in complex reflector geometries at a relatively low computational cost. A model is introduced that describes the received signal as the superposition of contributions from different geometrical areas with given characteristics in terms of signal phases. The local polynomial Fourier transform (LPFT) is then used to match the signal contributions that come from different image areas.

The second paper on image formation, by Wong et al., proposes a method of analysis for quantifying the image distortion introduced by the conventional Fourier transform approach. This analysis method involves a numerical model of the time-varying target rotation rate. The analysis implies that severe distortion is often attributed to phase modulation effects, whereas a time-varying Doppler frequency produces image smearing. Following insights gained from the analysis, the authors also propose a time-frequency processing/analysis based method for deblurring/refocusing conventionally generated ISAR images.

\section{Target classification and identification}

Radar signatures are often used for target classification and/or identification. The need for classifying a target has led to the development of high-resolution radar. ISAR images can be interpreted as two-dimensional (2D) radar signatures. Therefore, a 2D distribution of the energy backscattered from the target provides a multidimensional way of interpreting the information carried by the radar echo. Several techniques have been proposed for interpreting this ISAR-based information for the purpose of target classification/identification. These fall into two main philosophies: (i) feature matching and (ii) template or point matching, the latter being more oriented towards target identification. 
In this special issue, two papers deal with the problem of target classification by means of ISAR images. In the paper of Shreyamsha Kumar et al., a full system for target identification is proposed. The authors introduce a wavelet-based approach for ISAR image formation followed by feature extraction and target identification by means of neural networks. The use of the wavelet technique is compared with timefrequency techniques in terms of effectiveness and computational cost. In ISAR imaging it is sometimes difficult to predict the target orientation and often even more difficult to rescale the image along the cross-range coordinate. This problem is avoided in the proposed technique as the features used for target identification are invariant to translation, rotation, and scaling - leading to a robust ISAR image-based identification system.

The second paper by Radoi et al. proposes a supervised self-organising feature-based classification technique of super-resolution ISAR images. The super-resolution ISAR images are obtained through a MUSIC-2D method, coupled with phase unwrapping and symmetry enhancement. The proposed feature vector contains Fourier descriptors and moment invariants, which are extracted from the target shape and scattering center distribution of the ISAR image. These features, importantly, are invariant to target position and orientation. The feature-based classification is then carried out via a supervised adaptive resonance theory (SART) approach, which shows improved efficiency over the conventional MLP and fuzzy KNN classifiers.

Marco Martorella John Homer James Palmer Victor Chen Fabrizio Berizzi Brad Littleton Dennis Longstaff

Marco Martorella was born in Portoferraio (Italy) in June 1973. He received the Telecommunication Engineering Laurea and Ph.D. degrees from the University of Pisa (Italy) in 1999 and 2003, respectively. He became a postdoc. Researcher in 2003 and a permanent Researcher/Lecturer in 2005 at the Department of Information Engineering of the University of Pisa. He joined the Department of Electrical and Electronic Engineering (EEE) of the University of Melbourne during his Ph.D., the Department of Electrical and Electronic Engineering (EEE) of the University of Adelaide under a postdoc. contract, and the Department of Information Technology and Electrical Engineering (ITEE) of the University of Queensland as a Visiting Researcher between 2001 and 2006. His research interests are in the field of synthetic aperture radar (SAR) and inverse synthetic aperture radar (ISAR). He is an IEEE Member since 1999.
John Homer received the B.S. degree in physics from the University of Newcastle, Australia in 1985 and the Ph.D. degree in systems engineering from the Australian National University, Australia, in 1995. Between his B.S. and Ph.D. studies, he held a position of Research Engineer at Comalco Research Centre in Melbourne, Australia. Following his Ph.D. studies, he has held research positions with the University of Queensland, Veritas DGC Pty Ltd., and Katholieke Universiteit, Leuven, Belgium. He is currently a Senior Lecturer at the University of Queensland within the School of Information Technology and Electrical Engineering. His research interests include signal and image processing, particularly in the application areas of telecommunications, audio and radar. He is currently an Associate Editor of the Journal of Applied Signal Processing.

James Palmer was born in 1979 in Townsville, Australia. James received the Bachelor of electrical engineering (Hons I) and Bachelor of Arts (Japanese) degrees from the University of Queensland and is currently finishing his Ph.D. studies through the same institution. Palmer's major research interests are in the field of bistatic radar, SAR and ISAR (including the monostatic, emulated bistatic, and bistatic varieties), and sea surface forward scatter RF signal modelling and analysis.

Victor Chen received the Ph.D. degree in electrical engineering from Case Western Reserve University, Cleveland, Ohio, in 1989. Since 1990, he has been with Radar Division, the US Naval Research Laboratory in Washington DC and working on radar imaging, time-frequency applications to radar, ground moving target indication, and micro-Doppler analysis. He is a Principal Investigator working on various research

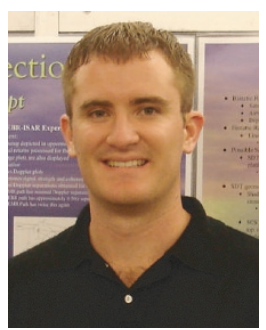
projects on radar signal and imaging, time-frequency applications to radar, and radar micro-Doppler effect. He served as Technical Program Committee Member and Session Chair for IEEE and SPIE conferences and served as a Guest Editor for IEE Proceedings on Radar, Sonar, and Navigation in 2003, and Associate Editor for the IEEE Trans. on Aerospace \& Electronic Systems since 2004. His current research interests include computational synthetic aperture radar imaging algorithms, micro-Doppler radar, and independent component analysis of features for noncooperative target identification. He received NRL Review Award in 1998, NRL Alan Berman Research Publication award in 2000 and 2004, and NRL Technical Transfer Award in 2002. He has more than 100 publications in books, journals, and proceedings including a book: Time-Frequency Transforms for Radar Imaging and Signal Analysis (V. C. Chen and Hao Ling), Artech House, Boston, Mass, January 2002.

Fabrizio Berizzi was born in Piombino (Italy) on November 1965 . He received the Electronic Engineering and Ph.D. degrees from the University of Pisa (Italy) in 1990 and 1994, respectively. Currently, he is an Associate Professor of the University of Pisa (Italy)_Department of Information Engineering. His main research interests are in the fields of synthetic aperture radar (SAR and ISAR), HF-OTH skywave and surface

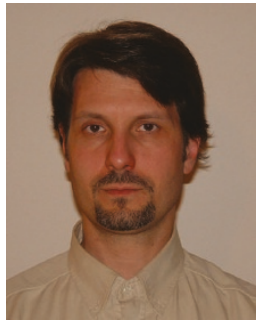


wave radar, target classification by wideband polarimetric radar data, hybrid waveform design for HRRP radar. He is the author and coauthor of more than 100 papers published in prestigious international journals, book chapters, and IEEE conference proceedings. $\mathrm{He}$ is the principal investigator of several research projects funded by Italian radar industries and by the Italian Minister of Defense. He cooperates to several research activities with the University of Adelaide (AUS), DSTO (AUS), JPL (USA), NRL (USA), ONERA (France), SOC (UK). He is a Member of the IEEE.

Brad Littleton received his Ph.D. in physics from the University of Queensland, in 2004. His research interests are elastic and inelastic electromagnetic wave/matter interactions, and applications to electromagnetic imaging, measurement and superresolution techniques. He is currently working on single quantum dot spectroscopy for the UQ node of the Centre for Quantum Computer Technology.

Dennis Longstaff is currently Technology Consultant to Filtronic PLC and Emeritus Professor with the School of Information Technology and Electrical Engineering at the University of Queensland. During that time at the University of Queensland, Dennis cofounded the Cooperative Research Centre for Sensor Signal and Information Processing (CSSIP). He was also
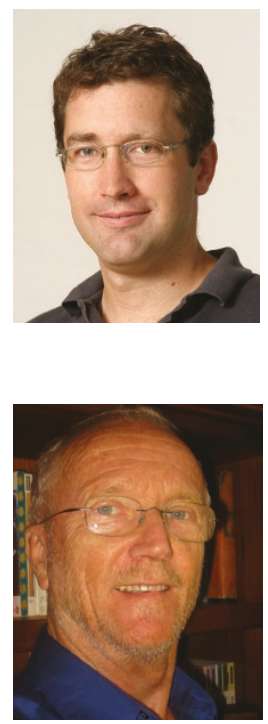
the Founder and Director of GroundProbe,

now a thriving global company marketing products invented by him and developed by his research group. He also served as Head of Department of Electrical and Computer Engineering for three years. From 1988 to 1991, he was at the Defence Science and Technology Organisation (DSTO) in Australia, where he was Research Leader to the Microwave Radar Division in Adelaide. Previous to this he spent 18 years as Senior Scientific Officer, then Principal Scientific Officer at the Royal Signals and Radar Establishment (now QintiQ), Malvern, England, where he worked on airborne radar systems. His work has attracted a number of awards and prizes and his spinoff company, GroundProbe, received an Engineering Excellence Award from the IE(Aust) Qld 2003. He was granted a Queensland Government Smart State Award in 2004, and an Australian Emerging Exporter Award in 2005 (see www.groundprobe.com). 